OPEN

SUBJECT AREAS:

MATERIALS PHYSICS

BIOMATERIALS

NANOBIOTECHNOLOGY

BIOPHYSICS

Received

9 May 2012

Accepted

20 June 2012

Published

16 July 2012

Correspondence and requests for materials should be addressed to J.L. (ijli@aphy.iphy.ac. cn) or C.G. (czgu@ aphy.iphy.ac.cn)

\section{Robust adhesion of flower-like few-layer graphene nanoclusters}

\author{
Shibing Tian' ${ }^{1}$ Lin Li', Wangning Sun' ${ }^{1}$ Xiaoxiang Xia' ${ }^{1}$, Dong Han², Junjie Li' \& Changzhi Gu'
}

${ }^{1}$ Beijing National Laboratory for Condensed Matter Physics, Institute of Physics, Chinese Academy of Sciences, Beijing 100190,
China, ${ }^{2}$ National Centre for NanoScience and Technology, Beijing 100190, China.

Nanostructured surface possessing ultrahigh adhesion like "gecko foot" or "rose petal" can offer more opportunities for bionic application. We grow flower-like few-layer graphene on silicon nanocone arrays to form graphene nanoclusters, showing robust adhesion. Their contact angle (CA) is $164^{\circ}$ with a hysteresis $\mathrm{CA}$ of $155^{\circ}$ and adhesive force for a $5 \mu \mathrm{L}$ water droplet is about $254 \mu \mathrm{N}$ that is far larger than present reported results. We bring experimental evidences that this great adhesion depends on large-area plentiful edges of graphene nanosheets tuned by conical nanostructure and intrinsic wetting features of graphene. Such new hierarchical few-layer graphene nanostructure provides a feasible strategy to understand the ultra-adhesive mechanism of the "gecko effect" or "rose effect" and enhance the wettability of graphene for many practical applications.

$\mathrm{n}$ nature, there are two particularly fascinating classes of adhesive phenomena: some plants like roses ${ }^{1,2} \mathrm{can}$ suspend a water droplet and keep it in a spherical shape on their petals and some animals, like certain species of gecko ${ }^{3-5}$ can selectively adhere to vertical surfaces, able to support far more than their body weight. In particular, the rose effect was once seen as paradoxical because of its coexisted properties of large contact angle (CA) and affinity to water ${ }^{6}$. As our related knowledge grows, this phenomenon has aroused great interest, both for fundamental research in surface wettability and for practical applications. And many procedures have been used in the ongoing effort to reveal the mechanism underlying this "rose petal effect." $2,7-12$ Some typical strategies of explaining for the rose effect include morphological factors ${ }^{2,11}$ composite micro-nanostructure ${ }^{7,9,10}$ chemical defects of hydrophobic surfaces ${ }^{8}$, and the triple-phase line (the solid-liquid-vapor line of contact) ${ }^{9,13}$. Although the mechanism remains unexplained, it is certain that nanostructure plays a key role in this perplexing phenomenon. Therefore, to design a nanostructure of some functional nanomaterials that has superior wettability is very helpful in further revealing and understanding the mechanism.

So far, this rose petal phenomenon has greatly inspired researchers to try to mimic experimentally the natural phenomenon for an extensive range of applications, such as dry adhesives and no-loss transport of liquid microdroplets $^{14-19}$. Typical among such research, L. Jiang's group ${ }^{2}$ has reported preparation of a polystyrene nanotube layer with high adhesive force and large CA, mimicking gecko's foot. In addition, other nanomaterials, such as carbon nanotubes ${ }^{15}, \mathrm{ZnO}$ nano pillars ${ }^{16}$ and $\mathrm{MnO}$ nanotubes ${ }^{20}$ have been used to form surfaces with high water adhesion and large $\mathrm{CA}^{16}$. Although adhesion force of about $130 \mu \mathrm{N}$ was obtained using a MnO nanomaterial ${ }^{20}$, science is still far from mastering the adhesive force and superhydrophobic surface for practical applications.

Recently, the wettability of graphene film has been widely studied ${ }^{21-29}$, and its strong hydrophobicity has been demonstrated in both theory and experiment. Theoretical results using density functional theory ${ }^{30}$ indicate that the binding energies between water molecules are larger than the associated adsorption energies on the graphene surface, explaining why water molecules form clusters on a graphene sheet. Experimental reports show that the contact angle (CA) of intrinsic graphene is of $127^{\circ 23}$. In addition, the higher adhesive property of graphene was also reported ${ }^{21,22}$, So the graphene may be a suitable candidate material to simultaneously realize large CA and ultrahigh adhesion. And some work has been done to study such properties of graphene ${ }^{25,29}$ by using thermal or chemical exfoliation of graphite flakes and depositing them on paper by suction filtration. But the morphology of films prepared this way may be hard to control and the flakes may be unstable for practical applications. A new method for fabrication of this kind of graphene surface is needed.

Toward this aim, we designed and fabricated a hierarchical structure of flower-like few-layer graphene nanosheets (GNSs) grown on silicon nanocone arrays (SNAs) to obtain super-wettability. The flower-like GNSs is synthesized by hot filament chemical vapor deposition, and SNAs are fabricated by maskless plasma etching. By taking advantage of silicon nanoconical structures, few-layer graphene can be grown on it into fullerblooming and better-dispersing nanosheets than can be synthesized on a planar surface. Thus, a dual roughness is 

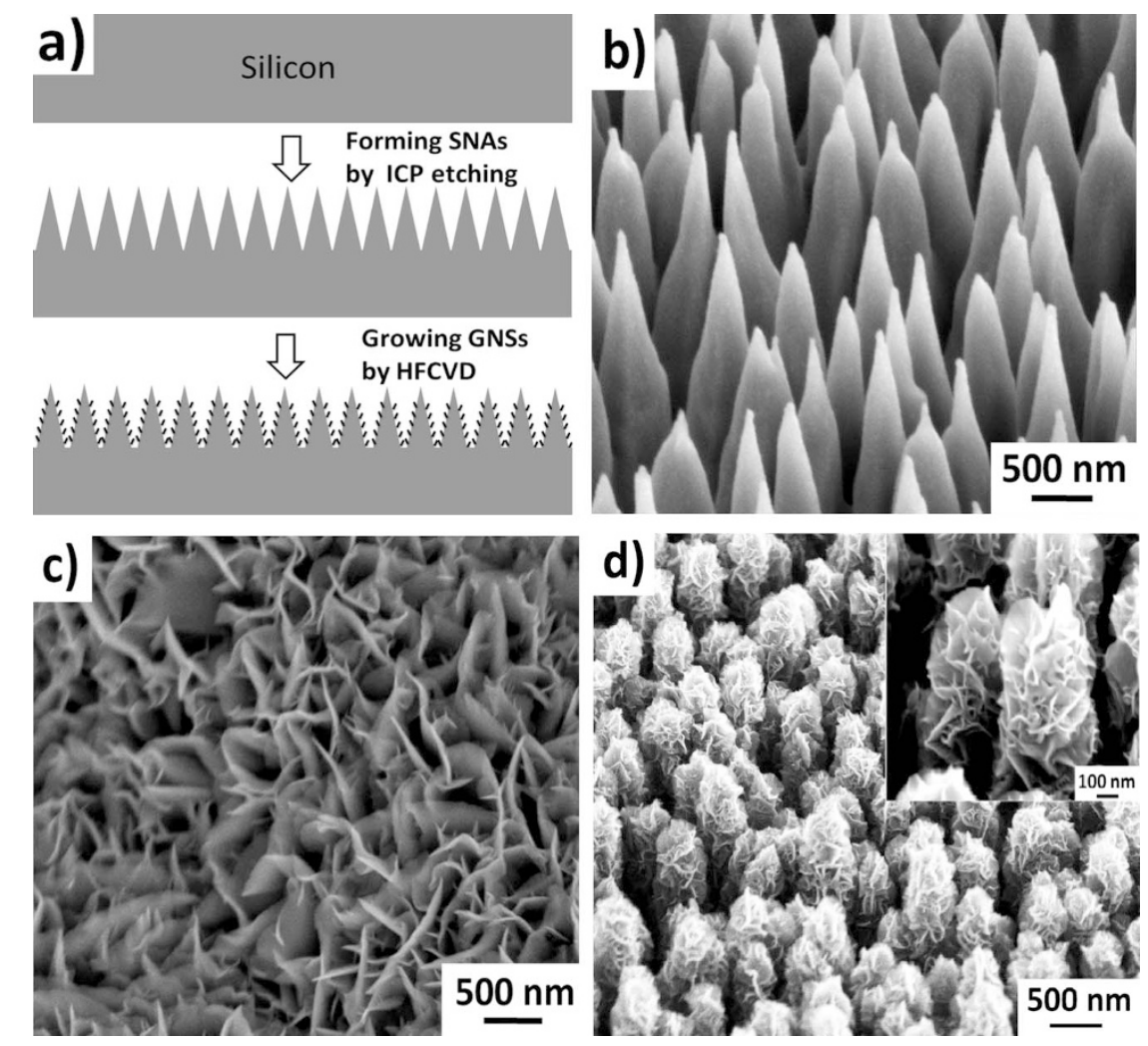

Figure $1 \mid$ (a) schematic illustration of the fabrication procedure of this hierarchical structure, (b) SEM image of SNAs, (c) SEM image of GNSs grown on polished surface, (d) SEM image of GNSs grown on SNAs; the inset shows high-magnification SEM image of GNSs grown on SNAs.

introduced by this design, which is favorable for enhancing the surface's wettability into a large CA state with ultrahigh adhesion. In addition, the effect of chemical modification and morphology tuning on hydrophobic and adhesive performance are explored. We find that the hydrophobic performance is similar with CA hysteresis, and the adhesive and hydrophobic properties can be improved synchronously by optimizing the hierarchical structure. Also, the mechanism behind such phenomenon is tentatively described. Our results provide a strategy to understand the ultra-adhesive mechanism of the "rose effect" and enhance the wettability of graphene for many practical applications.

\section{Results}

The hierarchical structures of GNSs combined with SNAs were fabricated by two simple steps schematically illustrated in Figure 1a). First, the fabrication of SNAs was realized in an Induction Coupling Plasma system ${ }^{31}$, as shown in Figure 1b). Geometric features of the SNAs have an apex diameter in the range $5 \sim 10 \mathrm{~nm}$, a base diameter of $\sim 300 \mathrm{~nm}$, lengths from $\sim 300$ to $800 \mathrm{~nm}$, and a tip density of $\sim 8 \times 10^{9} / \mathrm{cm}^{2}$. In the second step, the GNSs were synthesized by hot filament chemical deposition HFCVD. The morphology of the as-synthesized GNSs on polished silicon and SNAs respectively has also been characterized by scanning electron microscopy (SEM), as seen in Figure 1c) and d). It can be seen that the GNSs are uniform and perpendicular to the surface on both the polished silicon surface and the SNAs substrate. But, because of large surface area of the conical structure array, the GNSs grown on SNAs form fuller blooming structures than those grown on the polished silicon surface. This nano-textured surface will be fundamentally significant in understanding the film surface wetting property. More details of preparing samples were given in Method Section.

GNSs have been further characterized by transmission electron microscopy (TEM) and high-resolution TEM (HRTEM). Figure 2a) show the TEM images of single graphene growth vertically on the surface of SNAs. The plainly evident upward curling at the edges of the individual flakes might be due to internal stress in the few-layer graphene, and these edges make it possible to evaluate the thicknesses of the sheets. ${ }^{32}$ An HRTEM image of selected region $b$ of Figure 2a) is shown in Figure 2b) which shows that the number of layers in the graphene sheets is less than three. And an HRTEM images of selected region c of Figure 2a) is shown in Figure 2c), in which highly ordered lattice fringes can be observed, indicating that the GNSs are wellcrystallized. On the other hand, a TEM image of a perfect flowerlike GNSs is shown in Figure 2d) which reveals the flowerlike GNSs consist of a curled graphene nanoconical framework with scattered few-layer graphene sheets. An HRTEM image of selected region e of Figure 2d) is edge details of the flowerlike GNSs is shown in Figure 2e), which make it possible to evaluate the thicknesses of sheets at the areas indicated by arrows. Statistics of the sheets' thicknesses are shown in Figure 2f), based on examining 57 sections in several TEM images; this demonstrates that most of the GNSs are less than $4 \mathrm{~nm}$ thick.

The chemical composition and stoichiometry of the synthesized GNSs were further verified via Raman spectroscopy and electron energy loss spectroscopy (EELS) shown in Figure 2g) and h), respectively. The most prominent features in the Raman spectra of carbon materials are the so-called G band appearing at $1582 \mathrm{~cm}^{-1}$ and the D band at about $1350 \mathrm{~cm}^{-1}$. Besides, a symmetrical single $2 \mathrm{D}$ peak at around $2701 \mathrm{~cm}^{-1}$ is also observed, which stems from the second order of the zone-boundary phonons and is closely related to the number of layers in the graphene sheet. This $2 \mathrm{D}$ peak is more intense than the $G$ peak, indicating that the flower-like nanostructure is indeed highly organized few-layer graphene. The D peak is related to the disorders and defects of graphene ${ }^{33}$, and the intensity of the $\mathrm{D}$ peak relative to the $G$ peak $\left(I_{D} / I_{G}\right)$ reflects the abundance of defects in this graphene. The Raman spectrographic results of our sample indicate that the films contain $\mathrm{SP}^{2}$ carbon networks, but the disorder from defects is high, which is due both to the frequent defects in 

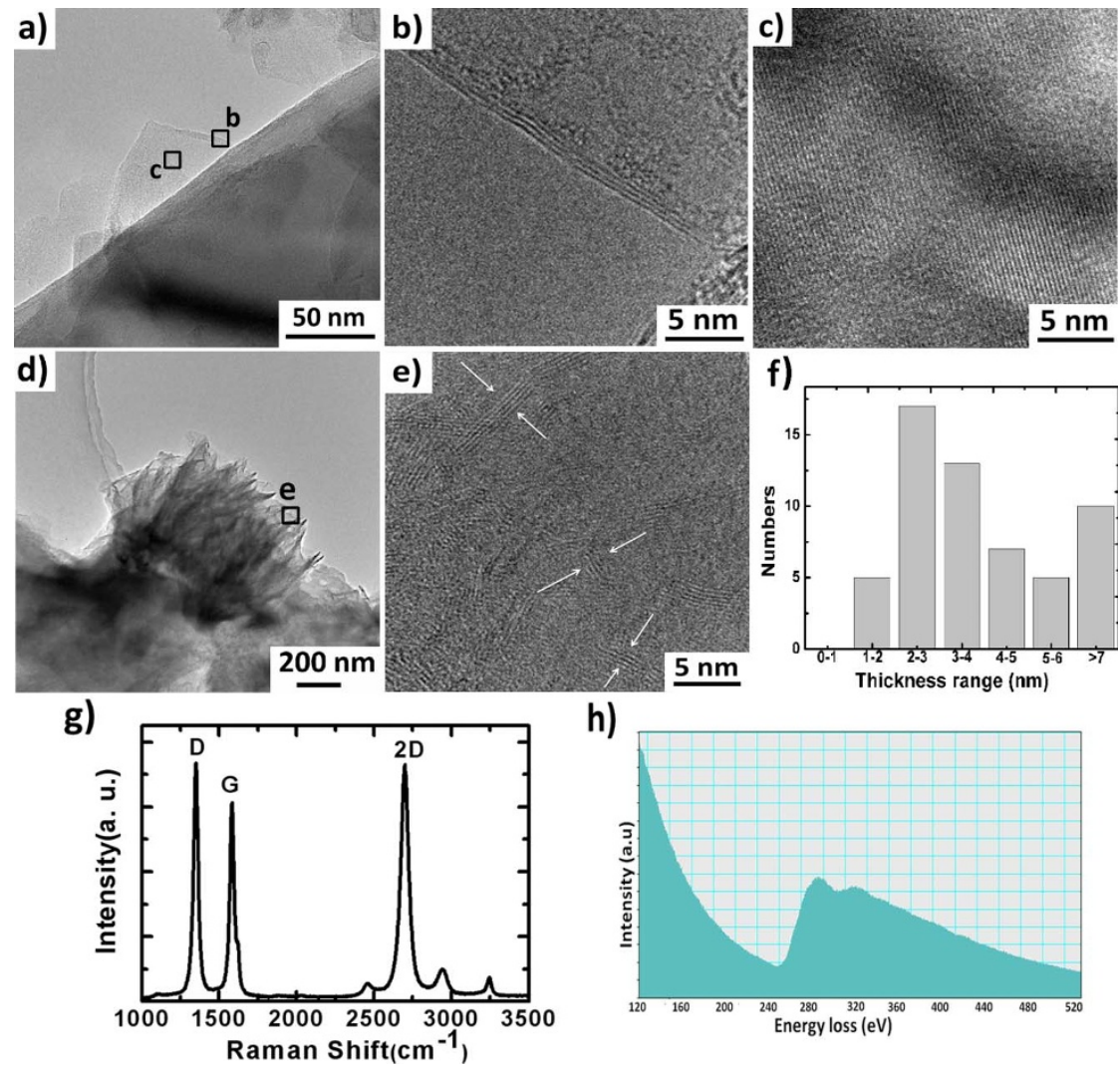

Figure 2 (a) Low-magnification TEM image of single graphene growth vertically on the surface of silicon nanocone. (b), (c), HRTEM images of the areas marked b, c in panel (a). (d) Low-magnification TEM image of a perfect flowerlike GNSs. (e) HRTEM images of the areas marked e in panel (d). (f) Statistics of the thickness distribution of GNSs. (g) Raman spectroscopy of GNSs. (h) Typical EELS spectrum of the GNSs.

the graphene film and to the numerous edges. Even so, most of the carbon atoms are in a highly oriented arrangement. The two main features of a graphite EELS spectrum in the carbon K-edge region are a peak at $285 \mathrm{eV}$ that corresponds to transitions from the 1 s to the $\pi^{*}$ states $\left(1 \mathrm{~s}-\pi^{*}\right)$, and a peak at $291 \mathrm{eV}$ that corresponds to transitions from the $1 \mathrm{~s}$ to the $\sigma^{*}$ states $\left(1 \mathrm{~s}-\sigma^{*}\right)^{34,35}$. The EELS spectrum for our GNSs (Figure $2 \mathrm{~h}$ )) also exhibited these characteristics. No trace of impurities is observed in the EELS spectrum. Based on the above mentioned characterizations, we judged that the GNSs were prepared successfully.

To investigate the wettability properties of as-formed surface characterized by the static CA, dynamic CA and adhesion force, the CA was measured using a Krüss DSA CA goniometer equipped with a dispensing needle. All the tests were performed in air at ambient temperature, and the CA value we report is the average of five measurements on the same sample. The biggest CA of our samples is about $164^{\circ}$ (shown in Figure 3a). From the Equation $(2.2 .1)^{36}$,

$$
\cos \theta=R_{f} f_{S L} \cos \theta_{0}-1+f_{S L},
$$

where $\theta$ and $\theta_{0}$ are the CA and original CA of a water droplet on the rough surface and flat surface respectively, $\mathrm{R}_{\mathrm{f}}$ is the roughness factor, $\mathrm{f}_{\mathrm{SL}}$ is the fraction of the solid-liquid contact area. The dual roughness of the surface is the main factor in enhancing the $\mathrm{R}_{\mathrm{f}}$ factor, leading to large CA. In addition, typical advancing angles $\left(\theta_{\mathrm{a}}\right)$ and receding angles $\left(\theta_{\mathrm{r}}\right)$ of as-formed surfaces are obtained by the dynamic sessile drop method, i.e., inflating and deflating the droplet volume, respectively, as shown in Figure $3 \mathrm{c}$ ) and d). A prime result shows that $\theta_{\mathrm{a}}$ and $\theta_{\mathrm{r}}$ values of our sample are $165^{\circ}$ and $10^{\circ}$. Thus, the CA hysteresis is $155^{\circ}$. Such high CA hysteresis can be explained by two factors: an adhesion hysteresis term $\Delta \mathrm{W}_{0}$ and an roughness term $\mathrm{H}_{\mathrm{r}}$, seen in Equation (2.2.2) ${ }^{9}$,

$$
\cos \theta_{a}-\cos \theta_{r}=\frac{\Delta W_{0}}{\gamma_{L V}}+H_{r},
$$

where $\theta_{\mathrm{a}}$ and $\theta_{\mathrm{r}}$ are the advancing CA and receding CA of a water droplet on the rough surface. Here, the work of adhesion of isolated graphene film is considerable, as previous reported, ${ }^{21}$ which contributes to this high CA hysteresis. Also, the roughness term $\mathrm{H}_{\mathrm{r}}$ is proportional to length of contact edges and density of silicon nanocones. It can be expressed by the Equation $(2.2 .3)^{9}$ :

$$
H r \propto \frac{D}{P^{2}},
$$

where $\mathrm{D}$ is the contact length per nanocone and $\mathrm{P}$ is the distance between neighboring nanocones. Here, the flowerlike GNSs provide numerous contact edges that make D extremely large, and the high density of SNAs can makes the P parameter very low. As a result, the high CA hysteresis is reasonable. Considering energy, if the triplephase line moves, it must overcome the potential barrier between metastable states. In this system, several strong forces are at play near the triple-phase line: the capillary force from nano-gaps among the GNSs and SNAs, the chemical affinity arising from the graphene defects revealed in the Raman spectra and the physical adhesion of numerous graphene edges. All three of these contribute to a high energy barrier between metastable states, i.e. a robust triple-phase line.

In addition, the high CA hysteresis value leads directly to the a high adhesive force ${ }^{7}$. Figure $3 \mathrm{~b}$ ) shows that a $5 \mu \mathrm{L}$ water droplet can be suspended on an upside down surface, and the inset shows the critical volume of water droplet hanging from the surface is $75 \mu \mathrm{L}$. And the slide angle of a $100 \mu \mathrm{L}$ water droplet on the verge of motion is about $70^{\circ}$ as can be seen in Figure $3 \mathrm{f}$ ). In this case, the value of the retention force is equal to the down slope gravitational force of magnitude $F_{r}=\rho V g \sin \alpha^{37}$, where $V$ is the drop volume, $\rho$ is the density of water 

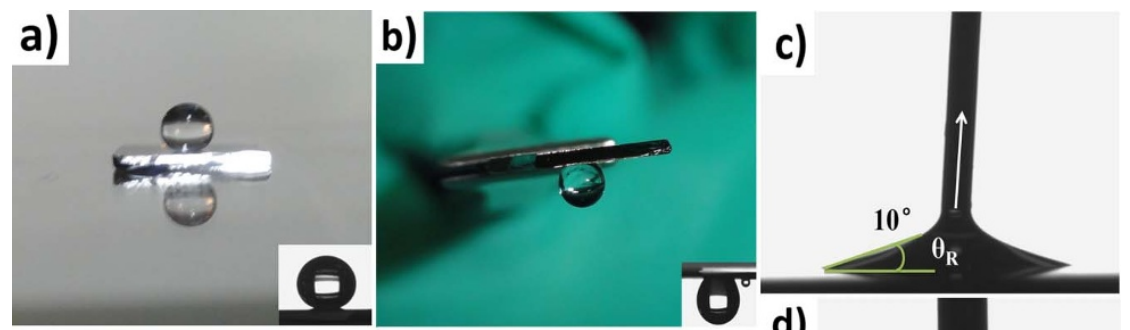

e)
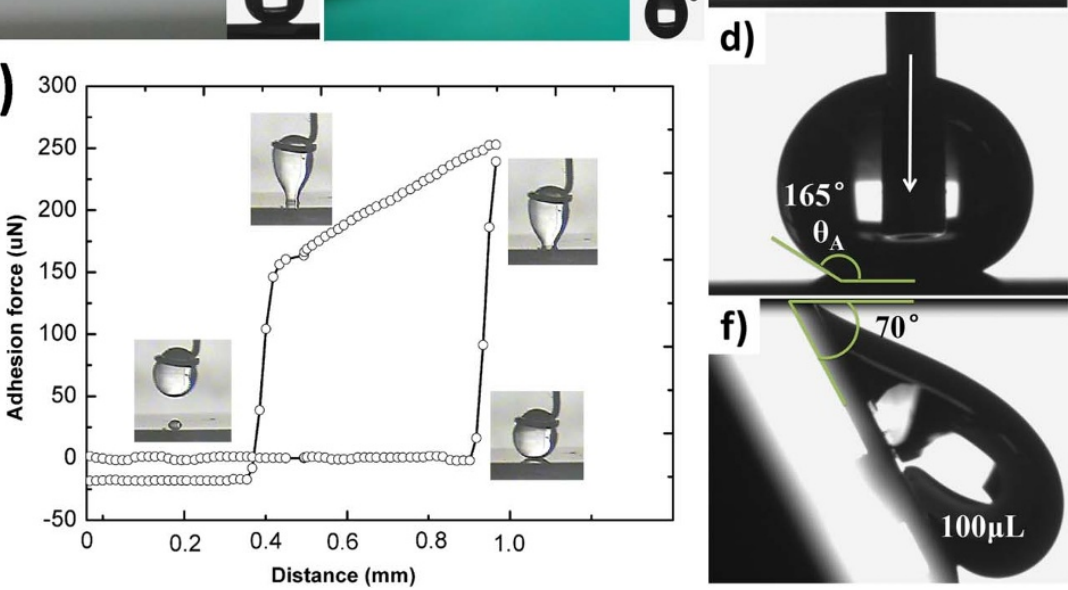

Figure $3 \mid$ (a) Shape of a water droplet on the sample's surface, indicating its large CA of $164^{\circ}$ (the inset is side view of $5 \mu \mathrm{L}$ water droplet). (b) Shape of water on the sample's surface when the sample is turned upside down (the inset is the side view of $75 \mu$ Lwater droplet). (c) Receding angle of water droplet when withdrawing water. (d) Advancing angle of water droplet when adding water. (e) Force-distance curves recorded before and after $5 \mu \mathrm{L}$ water droplet contacted the as-prepared hierarchical structure. (f) Slide angle of a $100 \mu \mathrm{L}$ water droplet on the verge of motion.

and $\mathrm{g}$ is the gravitational acceleration. Thus the $\mathrm{F}_{\mathrm{r}}$ value of this kind surface is about $700-900 \mu \mathrm{N}$. Furthermore, the adhesive action was assessed accurately by a high-sensitivity micro-electromechanical balance system, and detailed measurement process was given in Method

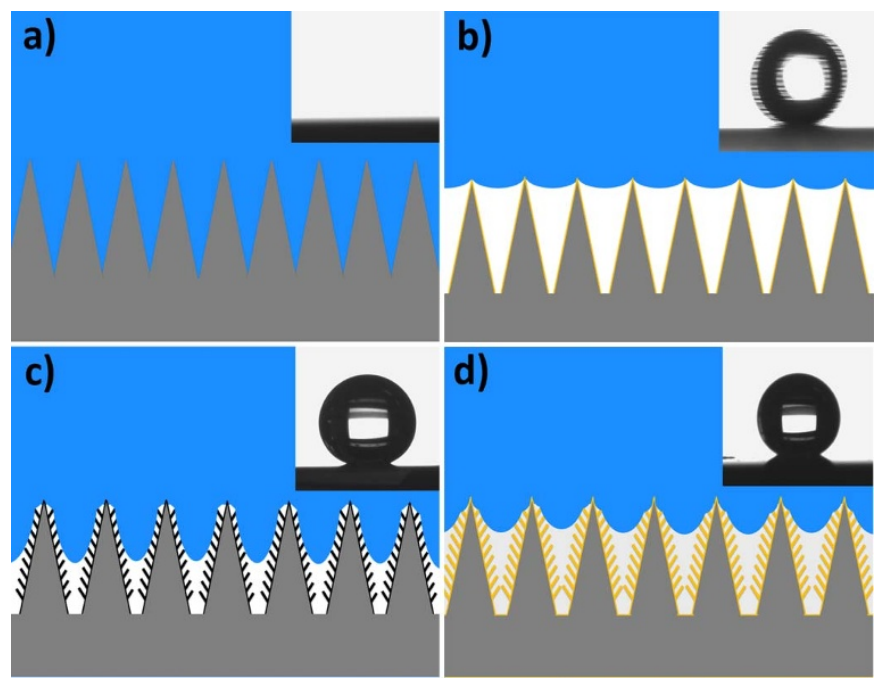

Figure $4 \mid$ Illustration of mechanism: (a) on the SNAs surface, water penetrates to the bases of the cones and spreads out (the inset is side view, after water contact); (b) on the SNAs surface modified with PFTES many air pockets exist between SNAs (the inset shows that a water droplet easily rolls away at very small tilt angle); (c) on GNSs/SNAs surface water cannot penetrate into the nano-gaps between the GNSs but does go partway into spaces between cones (the inset is side view of water CA $164^{\circ}$ ); (d) on the GNSs/SNAs surface modified with PFTES water also cannot penetrate into the nano-gaps between the GNSs, and it cannot go into spaces between cones as deeply as in unmodified samples (the inset is side view of water CA $\left.155^{\circ}\right)$.
Section. Figure 3e) displays the force-distance curves recorded before and after the water droplet contacted the structure with GNS-coated SNAs. It is observed that the surface has the very high adhesive force of $254 \mu \mathrm{N}$ for a $5 \mu$ Lwater droplet, which was nearly two times higher than the highest value reported so far $^{20}$.

We have shown that this GNSs/SNAs surface has unique wettability due to two factors: the morphological influence of this hierarchical structure and the material properties of GNSs. However, either factor independently influences the wettability remains unclear. To decouple the two effects, the samples were coated by self-assembled monolayer of $1 \mathrm{H}, 2 \mathrm{H}, 2 \mathrm{H}$-Perfluorodecyltriethoxysilane (PFTES) by gas-phase evaporation in a desiccator under vacuum for several hours, creating a surface with very low surface energy as reported earlier $^{38-40}$. The wettability of these samples was measured and the results are listed in Table 1 . It is notable that, after modified by PFTES, the wettability state of SNAs surface can be transformed from superhydrophilicity $\left(\mathrm{CA}<10^{\circ}\right)$ to superhydrophobicity $\left(\mathrm{CA}=172^{\circ}\right)$ with low adhesion about $10 \mu \mathrm{N}$. It is known that a silicon surface etched by oxygen plasma is hydrophobic due to numerous water affinitive chemical groups ${ }^{41}$. When a water droplet contacts the SNAs surface, it promptly penetrates to the bases of the cones and spreads out due to capillary action and disjoining pressure, as shown in Figure 4a). But, after modified with PFTES, the silicon cone's surface free energy becomes low and disjoining pressure becomes weak, making the cone repellent to water while the meniscus of water between cones becomes convex. Thus, water cannot enter into the space between silicon nanocones and many air pockets are packed under water, as seen in Figure 4b), which leads to less solid/water contact and a discontinuous triple-phase line. Therefore, the water droplet on the modified SNAs surface has larger CA and easy roll away at a very small tilt angle, as seen in the inset of Figure $4 \mathrm{~b}$ ).

However, for GNSs/SNAs surfaces, their water wetting process becomes more complex and different due to an introduction of vertical few-layer graphene nanosheets on the surfaces of silicon cones, and these graphene nanosheets define rich petal-like nanogaps between nanosheet-walls. Latest reports point out that the wetting 


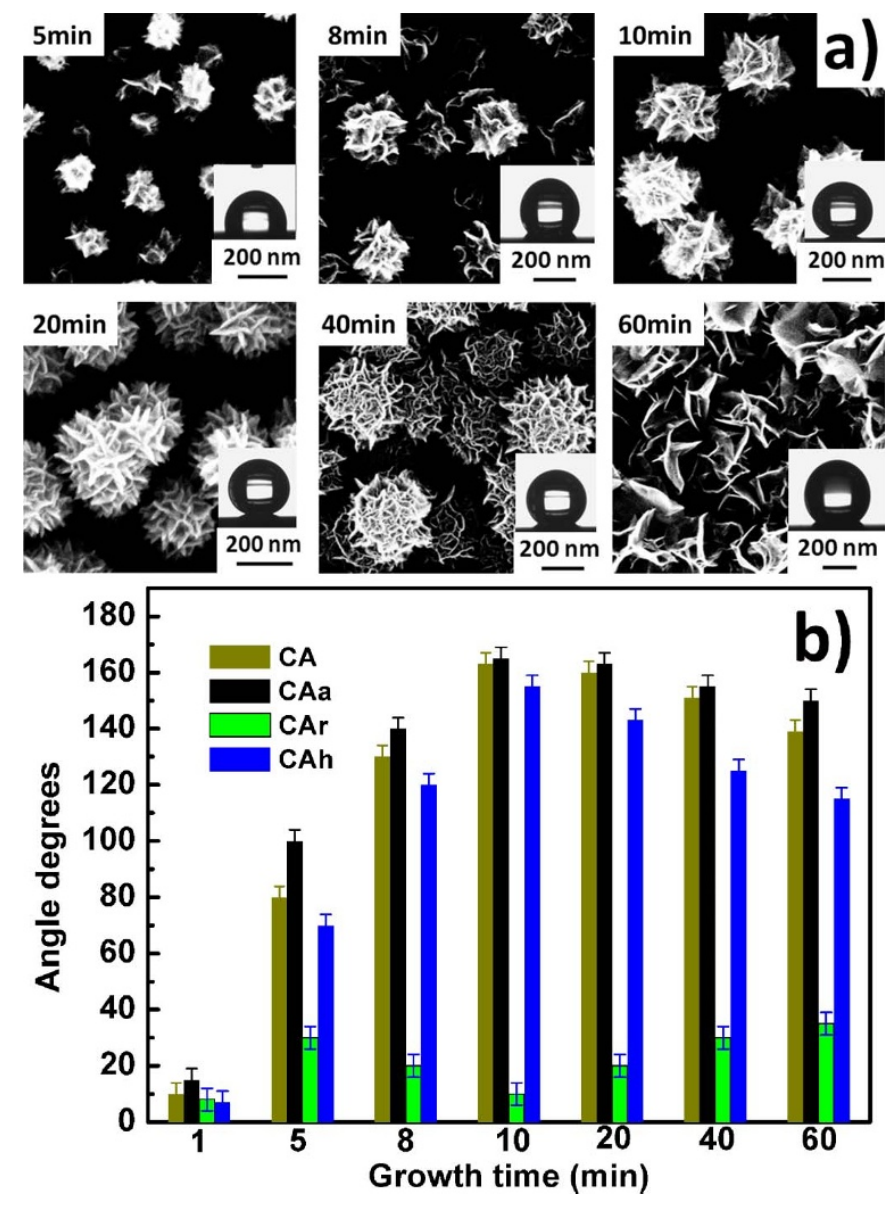

Figure $5 \mid$ (a) SEM images of GNSs coatings synthesized at $900^{\circ} \mathrm{C}$ for different durations, from 5 to $60 \mathrm{~min}$. Insets show optical images of a millimeter-sized water drop deposited with a syringe on corresponding surfaces. (b) The variation of CA, advancing CA (CAa), receding CA (CAr) and CA hysteresis (CAh) of hierarchical structures with GNSs and SNAs prepared with different growth times.

behavior of graphene mainly depends on its supporting substrate, due to graphene's extreme wetting transparency ${ }^{42}$. If this supporting substrate of graphene is air, the graphene is hydrophobic. In our experiment, few-layer graphene nanosheets stand in the air, and thus both sides of each nanosheet are hydrophobic. When water contacts GNSs/SNAs surfaces, it cannot penetrate between the nanosheets, which leads to a large CA of about $164^{\circ}$ as shown in the insets of Figure 4c). Here, the disjoining pressure effect may be very weak due to the hydrophobic nanostructures. This deduction is totally in accord with many previous reports that have directly observed in experiments that water can be repelled out of hydrophobic nanopores and nanocavities ${ }^{43-46}$. However, water still can partly enter these spaces between cones due to the affinity of plentiful graphene edges with high surface free energy, which leads to a high CA hysteresis of about $155^{\circ}$ and an adhesion force of about $254 \mu \mathrm{N}$, as drawn in Figure 4c). On the other hand, after the nanoclusters are modified with PFTES, the value of CA is lowered from $164^{\circ}$ to $155^{\circ}$ as shown in the insets of Figure d), and accordingly the value of CA hysteresis changes from $155^{\circ}$ to $96^{\circ}$ and adhesion force is $104 \mu \mathrm{N}$ weaker than that of unmodified GNSs/SNAs surfaces, as listed in table 1. The large decrease of CA hysteresis and adhesion can be explained by the fact that surface free energy of graphene edges is much lower after the graphene is modified with PFTES. In addition, water in contact with this modified surface cannot penetrate between the nanosheets as mentioned above, but water penetrates into spaces between cones less deeply than in unmodified samples due to a hydrophobic treatment by PFTES coating, as shown in Figure 4d). Thus, the triple phase line strength is weakened by the decreased CA, and the affinity of edges is partly suppressed due to lower CA hysteresis and adhesive force. These findings imply that if plenty of edges can be generated in nanoscale for water to contact, those edges will increase the overall adhesion of the larger scale surface. Further, our results above indicate that a great adhesion of $254 \mu \mathrm{N}$ is closely linked with the graphene edge's intrinsic properties, and the profuse defects induced by the abundant edges lead to high local surface energy and make an outstanding contribution to the graphene nanoclusters' high adhesion. Therefore, these distinct characteristics of the graphene edge play an important role in the ultrahigh adhesion of our GNSs/SNAs nanostructure's surface.

As discussed above, the hierarchical structure of GNSs/SNAs plays an important role in the wettability of such a surface. Here, we study morphology's effect on wettability. A series of GNSs with different morphology were synthesized through control of growth time. Their SEM images are shown in Figure 5a). Corresponding results of wettability tests are presented in Figure 5b), where it can be seen that the variability of CA is similar to that of CA hysteresis and that these values are lower for samples with either short growth time or long growth time. The GNSs' morphologies with 10-20 min growth time show the best wettability. This can be explained by the fact that with increasing growth time the GNSs grow more blooming. Thus, the surface roughness increases, which makes the CA become gradually bigger, and the numbers of graphene edges become more, which makes the triple line longer - i.e. increases CA hysteresis. However, when the nano-gaps of SNAs are filled little by little the effective GNSs will shrink to that like grown on a polished silicon surface. Then, these values deceased to a constant with the longer growth time. Thus, we determined that the optimal condition for synthesis of these morphologies was crucial to obtain ultra adhesive properties with large CA.

\section{Discussion}

We designed and fabricated a kind of hierarchical structure with flower-like GNSs coated on SNCs to mimic successfully the wettability "rose petal effect". This hierarchical structure shows CA larger than $160^{\circ}$, CA hysteresis of $155^{\circ}$ and ultrahigh adhesion to water, at about $254 \mu \mathrm{N}$ for a $5 \mu \mathrm{L}$ water droplet, which is far larger than previously reported results. By using chemical modification and morphological modulation, we find that the large CA of flower-like GNSs on the SNAs are due to the dual roughness of their hierarchical nanostructures. Further, their ultrahigh adhesion is mainly attributed to an integrated effect of plentiful nano-edges of GNSs and controllable nanogap between nanoclusters tuned by silicon nanocones. Among a great quantity of defects induced by these abundant edges lead to high local surface energy and hence make outstanding contribution to these GNS nanoclusters' high adhesion. In addition, unique intrinsic wetting features of graphene surface also play a positive role in increasing the adhesion of GNSs, which makes the triple line more robust. Therefore, both large and ultrahigh adhesion of material surface depends on the nanodetails of surface morphology and the material's intrinsic property, and flowerlike GNSs on the Si nancones possess both factors and hence show robust adhesion with large CA. Our design widens the practical function of related graphene nanostructures, and this chemically and mechanically stable surface might be useful for some devices such as 'lab on a chip' that need to hold and shunt around tiny quantities of liquid without leaking or being contaminated by nearby materials and for micro/ nanofluid handling applications.

\section{Methods}

First, the fabrication of SNAs was realized in an Induction Coupling Plasma (ICP) system (PlasmaLab System 100, Oxford Instruments). $\mathrm{SF}_{6}$ and $\mathrm{O}_{2}$ were utilized as etching gases, and a cryogenic temperature of $-110^{\circ} \mathrm{C}$ was maintained during the 
Table 1 Static CA, CA hysteresis and adhesive force of samples before and after chemical modification with PFTES

\begin{tabular}{lccc} 
Sample & Static CA & $\begin{array}{c}\text { CA hysteresis } \\
\Delta \theta=\theta_{A}-\theta_{R}\end{array}$ & $\begin{array}{c}\text { Adhesive } \\
\text { force }\end{array}$ \\
\hline SNAs & $<10^{\circ}$ & $*$ & $*$ \\
PFTES/SNAs & $172^{\circ}$ & $6^{\circ}=175^{\circ}-169^{\circ}$ & $10 \mu \mathrm{N}$ \\
GNSs/SNAs & $164^{\circ}$ & $155^{\circ}=165^{\circ}-10^{\circ}$ & $254 \mu \mathrm{N}$ \\
PFTES/GNSs/SNAs & $155^{\circ}$ & $96^{\circ}=161^{\circ}-65^{\circ}$ & $150 \mu \mathrm{N}$ \\
\hline *The values cannot be obtained on the superhydrophilic surface. & \\
\hline
\end{tabular}

etching process, as we reported elsewhere ${ }^{31}$. In the second step, the GNSs were synthesized by hot filament chemical deposition HFCVD. A mixed gas consisting of $4 \%$ methane and $96 \%$ hydrogen was used at a constant pressure of 20 Torr with a total gas flow of $100 \mathrm{sccm}$. This chemical reaction was activated by a tantalum filament $(8 \mathrm{~cm}$ in length and $0.5 \mathrm{~mm}$ in diameter) positioned at $8 \mathrm{~mm}$ above the substrate. The morphological and structural and chemical bond-state characterization of the samples obtained were carried out by scanning electron microscopy (SEM) image, transmission electron microscopy (TEM) images, high resolution transmission electron microscopy (HRTEM) images (at $200 \mathrm{kV}$ ), electron energy loss spectroscopy (EELS) and Raman spectra. The CA was measured using a Krüss DSA CA goniometer equipped with a dispensing needle. The sessile droplet was formed by fixing the needle and approaching the substrate parallel to the needle direction with a very gentle feed rate of a few micrometers per minute. All the tests were performed in air at ambient temperature. The adhesive action was assessed accurately by a highsensitivity micro-electromechanical balance system. First, the sample surface was placed on the plate of a balance system, a $5 \mu \mathrm{Lwater}$ droplet was suspended on a metal ring, and the force of the balance system was initialized to zero. Then, the sample surface was brought into contact with the droplet while maintaining the balance force at zero. The surface was moved at a rate of $0.02 \mathrm{~mm} \mathrm{~s}^{-1}$. When the surface left the water droplet after contact, the balance force increased gradually and reached its maximum. Finally, the balance force decreased immediately when the surface broke away from the droplet to finish one cycle of the measurement.

1. Kaplan, M. Raindrops on roses. Nature News, doi:10.1038/news.2008.778 (2008).

2. Feng, L. et al. Petal effect: a superhydrophobic state with high adhesive force. Langmuir 24, 4114-4119 (2008).

3. Geim, A., Grigorieva, S. V. D. I. V., Novoselov, K., Zhukov, A. \& Shapoval, S. Y. Microfabricated adhesive mimicking gecko foot-hair. Nature Materials $\mathbf{2}$, 461-463 (2003).

4. Autumn, K. et al. Evidence for van der Waals adhesion in gecko setae. Proceedings of the National Academy of Sciences 99, 12252-12256 (2002).

5. Huber, G. et al. Evidence for capillarity contributions to gecko adhesion from single spatula nanomechanical measurements. Proceedings of the National Academy of Sciences of the United States of America 102, 16293-16296 (2005).

6. Li, W. \& Amirfazli, A. Superhydrophobic surfaces: adhesive strongly to water? Advanced Materials 19, 3421-3422 (2007).

7. Nosonovsky, M. \& Bhushan, B. Lotus Versus Rose: Biomimetic Surface Effects. Green Tribology, 25-40 (2012).

8. Chang, F. M., Hong, S. J., Sheng, Y. J. \& Tsao, H. K. High contact angle hysteresis of superhydrophobic surfaces: Hydrophobic defects. Appl. Phys. Lett. 95 064102-064103 (2009).

9. Nosonovsky, M. Model for solid-liquid and solid-solid friction of rough surfaces with adhesion hysteresis. J Chem Phys 126, 224701 (2007).

10. Bhushan, B. \& Her, E. K. Fabrication of superhydrophobic surfaces with high and low adhesion inspired from rose petal. Langmuir 26, 8207-8217 (2010).

11. Liu, M. J. \& Jiang, L. Switchable Adhesion on Liquid/Solid Interfaces. Advanced Functional Materials 20, 3753-3764 (2010).

12. Bormashenko, E., Stein, T., Pogreb, R. \& Aurbach, D. "Petal Effect" on Surfaces Based on Lycopodium: High-Stick Surfaces Demonstrating High Apparent Contact Angles. The Journal of Physical Chemistry C 113, 5568-5572 (2009).

13. Gao, L. \& McCarthy, T. J. Contact angle hysteresis explained. Langmuir 22, 6234-6237 (2006).

14. Lee, H., Lee, B. P. \& Messersmith, P. B. A reversible wet/dry adhesive inspired by mussels and geckos. Nature 448, 338 (2007).

15. Sethi, S., Ge, L., Ci, L., Ajayan, P. M. \& Dhinojwala, A. Gecko-inspired carbon nanotube-based self-cleaning adhesives. Nano Letters 8, 822-825 (2008).

16. Li, J., Liu, X. H., Ye, Y. P., Zhou, H. D. \& Chen, J. M. Gecko-inspired synthesis of superhydrophobic $\mathrm{ZnO}$ surfaces with high water adhesion. Colloid Surface A 384, 109-114 (2011).

17. Hu, S., Jiang, H., Xia, Z. \& Gao, X. Friction and adhesion of hierarchical carbon nanotube structures for biomimetic dry adhesives: multiscale modeling. ACS Appl Mater Interfaces 2, 2570-2578 (2010).

18. Cho, W. K. \& Choi, I. S. Fabrication of hairy polymeric films inspired by geckos: wetting and high adhesion properties. Advanced Functional Materials 18, 1089-1096 (2008)
19. Northen, M. T. \& Turner, K. L. A batch fabricated biomimetic dry adhesive. Nanotechnology 16, 1159 (2005).

20. Zhao, X. D. et al. Electrically Adjustable, Super Adhesive Force of a Superhydrophobic Aligned MnO2 Nanotube Membrane. Advanced Functional Materials 21, 184-190 (2011).

21. Huang, R. Graphene: Show of adhesive strength. Nature Nanotechnology 6 537-538 (2011).

22. Koenig, S. P., Boddeti, N. G., Dunn, M. L. \& Bunch, J. S. ultra strong adhesion of graphene membranes. Nature Nanotechnology 6, 543-546 (2011).

23. Wang, S., Zhang, Y., Abidi, N. \& Cabrales, L. Wettability and surface free energy of graphene films. Langmuir 25, 11078-11081 (2009).

24. Kou, L. \& Gao, C. Making silica nanoparticle-covered graphene oxide nanohybrids as general building blocks for large-area superhydrophilic coatings. Nanoscale 3, 519-528 (2010).

25. Rafiee, J., Rafiee, M. A., Yu, Z. Z. \& Koratkar, N. Superhydrophobic to superhydrophilic wetting control in graphene films. Advanced Materials 22, 2151-2154 (2010).

26. Hong, J. \& Kang, S. W. Hydrophobic properties of colloidal films coated with multi-wall carbon nanotubes/reduced graphene oxide multilayers. Colloids and Surfaces A: Physicochemical and Engineering Aspects 374, 54-57 (2011).

27. Hsieh, C. T. \& Chen, W. Y. Water/oil repellency and work of adhesion of liquid droplets on graphene oxide and graphene surfaces. Surface and Coatings Technology 205, 4554-4561 (2011).

28. Lin, Y., Ehlert, G. J., Bukowsky, C. \& Sodano, H. Superhydrophobic Functionalized Graphene Aerogels. ACS Appl. Mater. Interfaces 3, 2200-2203 (2011).

29. Zhang, X., Wan, S., Pu, J., Wang, L. \& Liu, X. Highly hydrophobic and adhesive performance of graphene films. J. Mater. Chem. 21, 12251-12258 (2011).

30. Leenaerts, O., Partoens, B. \& Peeters, F. Water on graphene: Hydrophobicity and dipole moment using density functional theory. Physical Review B 79, 235440 (2009).

31. Tian, S. et al. Large-scale ordered silicon microtube arrays fabricated by Poisson spot lithography. Nanotechnology 22, 395301 (2011).

32. Malesevic, A. et al. Synthesis of few-layer graphene via microwave plasmaenhanced chemical vapour deposition. Nanotechnology 19, 305604 (2008).

33. Pimenta, M. et al. Studying disorder in graphite-based systems by Raman spectroscopy. Phys. Chem. Chem. Phys. 9, 1276-1290 (2007).

34. Dato, A., Radmilovic, V., Lee, Z., Phillips, J. \& Frenklach, M. Substrate-free gasphase synthesis of graphene sheets. Nano Letters 8, 2012-2016 (2008).

35. Panchakarla, L. et al. Synthesis, Structure, and Properties of Boron-and NitrogenDoped Graphene. Advanced Materials 21, 4726-4730 (2009).

36. Cassie, A. B. D. \& Baxter, S. Wettability of porous surfaces. T Faraday Soc $\mathbf{4 0}$, 0546-0550 (1944).

37. Chu, K. H., Xiao, R. \& Wang, E. N. Uni-directional liquid spreading on asymmetric nanostructured surfaces. Nat Mater 9, 413-417 (2010).

38. Hoffmann, P. W., Stelzle, M. \& Rabolt, J. F. Vapor phase self-assembly of fluorinated monolayers on silicon and germanium oxide. Langmuir 13 1877-1880 (1997).

39. Wang, T., Isimjan, T. T., Chen, J. \& Rohani, S. Transparent nanostructured coatings with UV-shielding and superhydrophobicity properties. Nanotechnology 22, 265708 (2011).

40. Kölbel, M. et al. Self-Assembled Monolayer Coatings on Nanostencils for the Reduction of Materials Adhesion. Advanced Functional Materials 13, 219-224 (2003).

41. Zhou, Y., Wang, X., Guo, X., Qiu, X. \& Liu, L. A water collecting and recycling structure for silicon-based micro direct methanol fuel cells. International Journal of Hydrogen Energy 37, 967-976 (2012).

42. Rafiee, J. et al. Wetting transparency of graphene. Nature Materials 11, 217-222 (2012).

43. Chen, P. et al. Wetting Behavior at Micro-/Nanoscales: Direct Imaging of a Microscopic Water/Air/Solid Three-Phase Interface. Small 5, 908-912 (2009).

44. Chen, P., Jiang, L. \& Han, D. In Situ Imaging of Multiphase Bio-interfaces at the Micro-/Nanoscale. Small 7, 2825-2835 (2011).

45. Lum, K., Chandler, D. \& Weeks, J. D. Hydrophobicity at small and large length scales. The Journal of Physical Chemistry B 103, 4570-4577 (1999).

46. Powell, M. R., Cleary, L., Davenport, M., Shea, K. J. \& Siwy, Z. S. Electric-fieldinduced wetting and dewetting in single hydrophobic nanopores. Nature Nanotechnology 6, 798-802 (2011).

\section{Acknowledgements}

We thank Prof. Shutao Wang for helpful discussion. This work is supported by the National Natural Science Foundation of China (Grand No. 91023041, 50825206, 10834012,11174362), the National Basic Research Program of China (Grand No.2009CB930502) and the Knowledge Innovation Project of CAS (Grand No. KJCX2-EW-W02).

\section{Author contributions}

C.Z. Gu and J.J. Li planned and coordinated the experiments. L. Li, W.N. Sun, S.B. Tian and X.X. Xia fabricated the Si nanocone arrays and grew GNSs, and then performed SEM and TEM measurement. S.B. Tian carried out the experiments of samples' wettability properties, 
and D. Han and C.Z. Gu helped with the data discussion and interpretation, S.B. Tian and J.J. Li wrote the paper.

\section{Additional information}

Competing financial interests: The authors declare no competing financial interests.
License: This work is licensed under a Creative Commons

Attribution-NonCommercial-ShareAlike 3.0 Unported License. To view a copy of this license, visit http://creativecommons.org/licenses/by-nc-sa/3.0/

How to cite this article: Tian, S. et al. Robust adhesion of flower-like few-layer graphene nanoclusters. Sci. Rep. 2, 511; DOI:10.1038/srep00511 (2012). 